\title{
Computational Design and Motion Control for Characters in the Real World
}

\author{
Stelian Coros \\ Disney Research Zurich
}
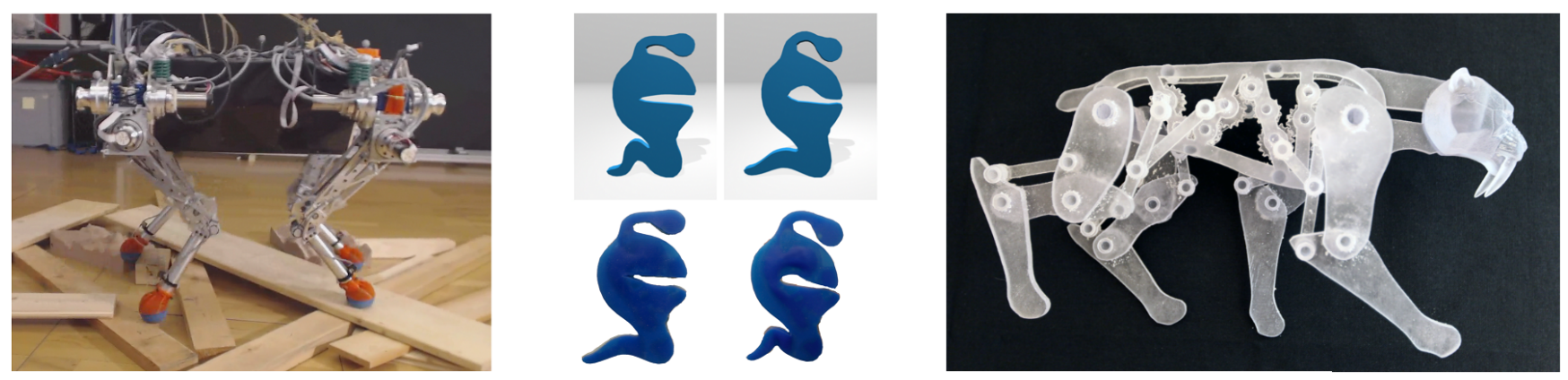

Figure 1: Examples of real-world characters: an autonomous dog-like robot capable of traversing challenging environments; squishy digital characters converted into fabricated prototypes that deform in prescribed ways; $3 D$ printed mechanical characters designed to produce user-specified motions.

\begin{abstract}
Computer graphics techniques allow artists to realize their imaginative visions, leading to immersive virtual worlds that capture the imagination of audiences world-wide. And now, thanks to advancements in rapid manufacturing devices, tangible links between these vivid virtual worlds and our own can be created. But in order to unleash the full potential of this technology, a key challenge lies in determining the fundamental principles and design paradigms that allow digital content to be processed into forms that are suitable for fabrication. A particularly challenging task is that of creating physical representations of animated virtual characters. This paper discusses several techniques that can be applied towards this goal. In particular, a method for controlling the deformation behavior of real-world objects is described, and a computational design system that allows casual users to create animated mechanical characters is presented. In addition, this paper shows that control algorithms developed for physics-based character animation can also be applied to legged robots, allowing them to move with skill and purpose.
\end{abstract}

CR Categories: I.3.7 [Computer Graphics]: Three-Dimensional Graphics and Realism-Animation;

Keywords: mechanical characters, animation, computational design, robotics, control

Links: DL $₫ \mathrm{PDF}$

\section{Introduction}

A key factor in the appeal of today's movies and video-games is that the virtual worlds they portray place no limits on what can be imagined. Within these worlds, alien creatures and environments can easily be brought to life, for instance. Rapid manufacturing devices hold the promise of bringing this type of freedom to our own world by allowing us to fabricate physical objects whose appearance, deformation behaviors and motions can be specified and controlled as intuitively as when creating assets for virtual worlds. In order to unleash the full potential of this technology, however, computational design methods that create digital content suitable for fabrication need to be developed. A number of research projects aimed at this problem are starting to appear in the literature. For instance, automated methods for creating poseable representations of video-game characters have been proposed [Bächer et al. 2012; Calì et al. 2012], and Prevost et al. [2013], showed that by adapting the shape and material distribution, it is possible to design and 3D print characters that can balance statically. Fabricating static embodiments of virtual characters, however, is just the beginning.

In virtual environments, animated characters are key to creating compelling visual experiences. Robots and animatronic figures play an equivalent role in theme parks, and mechanical toys that exhibit interesting movements are popular consumer products. The design and motion repertoires for such real-world characters, unlike their virtual counterparts, are strictly bound by real-world constraints. A long term challenge then is to automate the process of designing, fabricating and animating real-world characters so that they can match the fluidity and grace that is expected of characters in video games and CG movies. This paper outlines three recent approaches that were developed with the goal of bringing animated virtual characters to the real world.

\section{Computational Design of Animated Me- chanical Characters}

The first challenge that needs to be overcome in order to bring virtual characters to the real world is that of designing their mechanical structure. This task is currently restricted to skilled and experienced engineers, who, given sufficient time and resources, can design complex robots, mechanical toys or animatronic figures 
capable of moving with life-like fluidity. As a first step towards automating this process, we recently introduced a computational design system that allows non-expert users to create complex mechanical characters whose motions are driven by one actuator or a manually-operated crank [Coros et al. 2013].
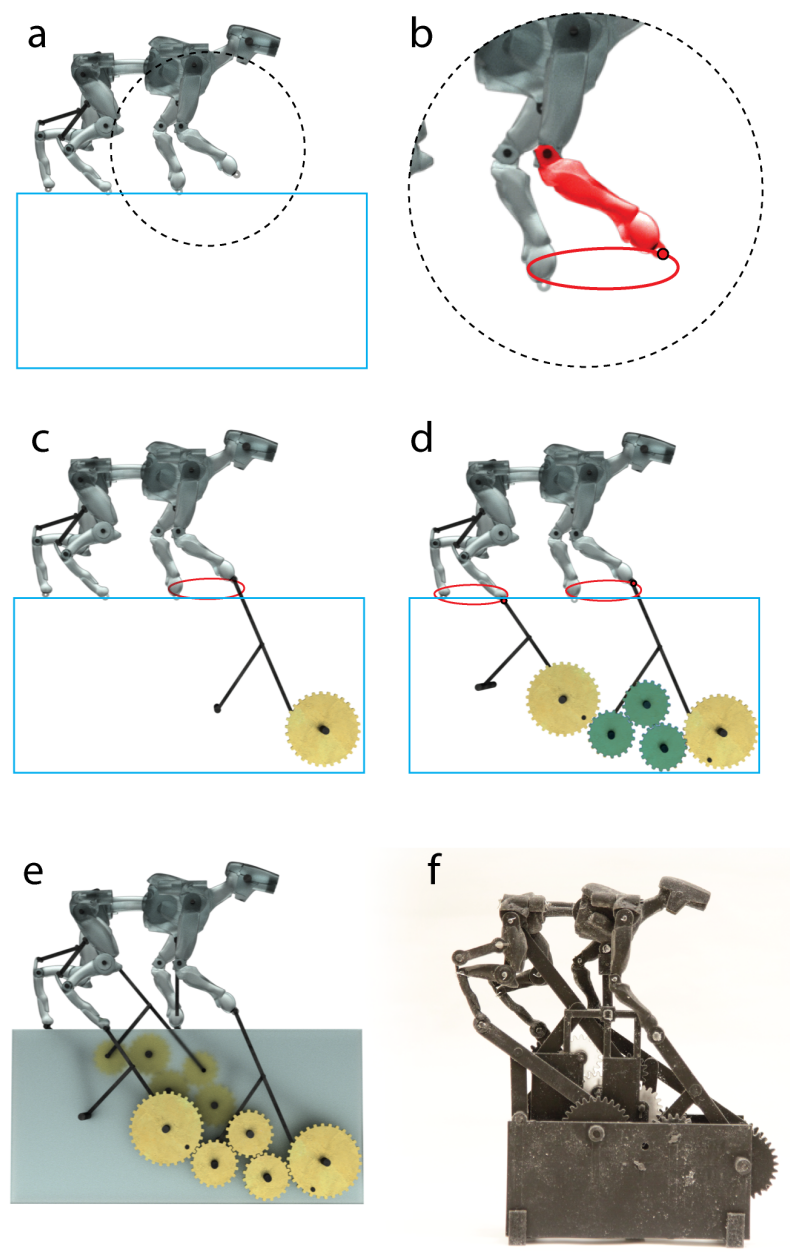

Figure 2: Overview of our computational design system for mechanical characters: a) an input character is provided, and the relative position of the gear box is specified; $b$ ) the desired motion of the character is specified by sketching motion curves; c) optimized driving mechanisms are generated by our framework; d) gear trains are created to connect all the driving mechanisms to one actuator; e) support structure is generated; $f$ ) the resulting mechanical character is 3D-printed.

Our system assumes access to a set of different types of mechanical assemblies that are to be used as building blocks. Each type of assembly is parameterized, and, in an offline step, our system performs a sparse sampling of their parameter spaces. This precomputation step results in a database of representative motions that each type of mechanical assembly can perform. After this offline step, our computational design system allows casual users to interactively create mechanical characters.

As illustrated in Figure 2, the desired motion of an input articulated character is specified through motion curves provided for a selected set of actuation points. For each actuation point, our system computes an optimized mechanical assembly that reproduces its desired motion trajectory as closely as possible. This happens in a two-stage process. First, the precomputed database is queried, returning both the type of mechanical assembly that is best suited for each motion curve, as well as a good set of parameter values for it. Since the database only stores a sparse sampling of the parameter spaces for each type of mechanism, however, the retrieved assemblies are not likely to be optimal. They are therefore further optimized using a gradient-based method. Because the set of parameters obtained from the database look-up already provides a good starting point, the continuous optimization method converges quickly and bad local minima can be avoided.

The resulting optimized mechanical assemblies are instantiated and connected to the character using pin joints, driving its motions as specified by the user. The gears that actuate the individual driving assemblies are then connected to each other through a gear train in a user-guided, semi-automatic fashion. For planar characters, our system can automatically reposition the mechanical components to ensure that no collisions occur as the character is animated, and finally, support structures are generated to hold the components of the assembly in place. The mechanical character and the assembly that controls its motion are then ready to be fabricated.

We demonstrate the versatility of our computational design system by creating a wide range of mechanical characters, seven of which we manufactured using $3 \mathrm{D}$ printing.

\section{Computational Design of Deformable Char- acters}

While some virtual characters are rigidly articulated, others are inherently deformable, and their motions reflect and exploit these characteristics. When creating physical embodiments of such virtual characters, therefore, the ways in which they deform should be taken into account and reproduced as closely as possible. By analyzing the equations of motion of elastic objects, it becomes clear that controlling their deformation behavior can be achieved in several different ways: by adapting the rest, or undeformed, state of the objects, by changing the material properties governing the relation between deformations and internal forces, or by explicitly applying external forces. In simulation, the process of automatically adapting the rest state of deformable objects in order to control their motions works well [Coros et al. 2012]. However, the technology that allows fabricated objects to significantly change their rest configuration in a controllable manner is currently unavailable, so we must resort to using external forces and modulating material properties.

In recent work, we introduced a method that automates the process of creating physical replicas of deformable virtual characters [Skouras et al. 2013]. We take as input a deformable character and a set of target poses for it, as illustrated in Figure 3. Our method combines finite-element analysis, sparse regularization and constrained optimization in order to compute a minimal set of actuation points along the character's surface where external forces should be applied, as well as an optimized, inhomogeneous distribution of material properties. This output can be used to fabricate physical characters whose range of deformations closely matches the target shapes that were provided as input. To validate our framework, we applied our pipeline on a set of $2 \mathrm{D}$ and $3 \mathrm{D}$ characters that are both simulated and physically-fabricated.

\section{Motion Control}

Once the mechanical structure of real-world characters is designed and fabricated, the remaining task is to control their motions. For under-actuated systems with many active degrees of freedom, such as human or animal-like robots, this is notoriously difficult: gen- 


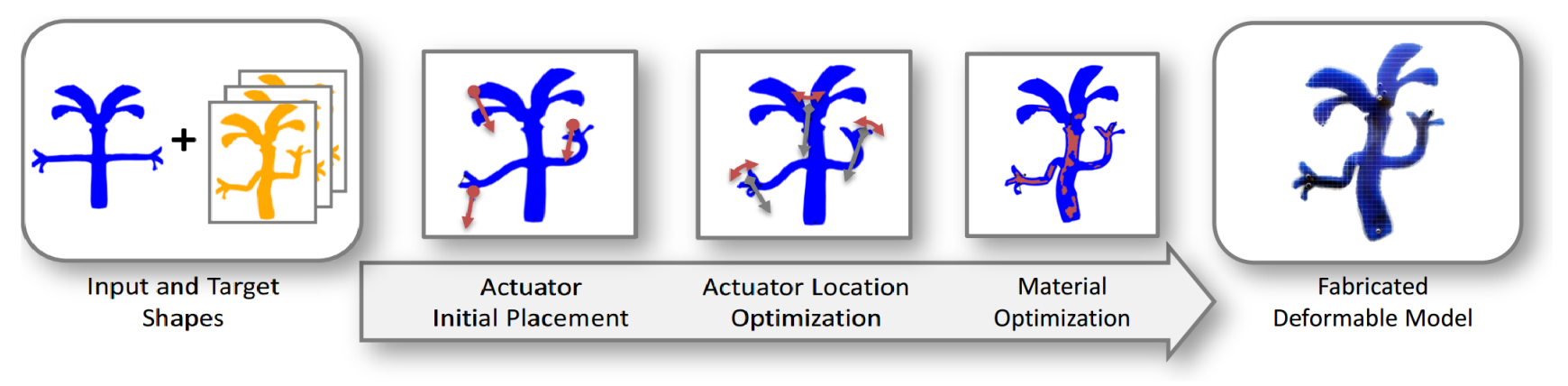

Figure 3: Steps to designing deformable characters: an input character and target shapes are taken as input; a small number of actuators and their initial placements are determined; the positions of the actuators and the internal material distribution are optimized; the physical prototype is fabricated.

erating even basic motions requires a tremendously complex interplay of sensory information processing, motion planning, and coordinated control signals for the available actuators. The challenges associated with this problem have been studied for more than two decades, both in the context of robotics, and from the point of view of character animation. Generally speaking, the overarching goal of this effort is to understand and reproduce the mathematical, biomechanical and motor-learning principles that allow humans and animals to move with skill, grace and agility.

Over the past few years, we have made significant progress on this challenging problem by developing control solutions that allow physically-simulated characters to walk and run robustly [Coros et al. 2010; Coros et al. 2011]. In subsequent work, by performing experiments on StarlETH [Hutter et al. 2012], we have shown that the same control strategies can be applied equally well to legged robots [Gehring et al. 2013]. A factor key to the success of our control solution is that it is designed to handle significant unanticipated perturbations (variations in the terrain, pushes, sensor or actuator noise, etc), and it therefore does not over-fit to the simulation environment it was initially developed for.
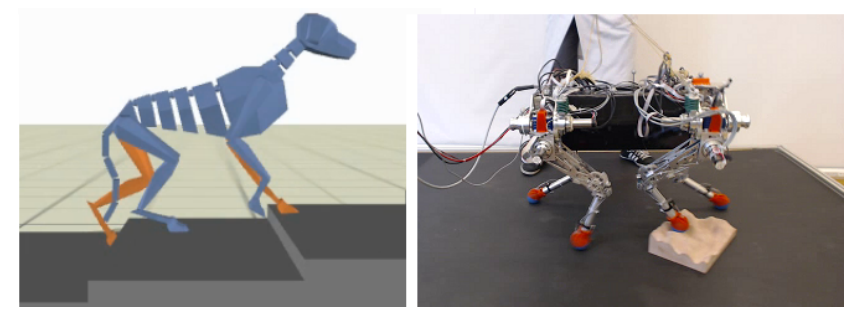

Figure 4: Locomotion control strategies allow both physicallysimulated creatures and legged robots to walk robustly.

The control scheme we employ combines several simple building blocks: an inverted pendulum model computes desired foot fall locations, proportional-derivative controllers regulate the motions of the legs during swing, and virtual forces applied through the stance legs are used to continuously modulate the position and orientation of the main body. We formulate the task of computing appropriate virtual forces for each stance leg as a constrained optimization problem which is solved at every control step. The result is an optimal distribution of virtual forces that ensures the resulting joint torques are within reasonable limits and that the feet do not slip. We demonstrate the flexibility of the described framework by generating walking and trotting controllers that are robust to pushes and unanticipated variations in the terrain, as shown in Figure 4.

\section{Conclusion}

Given the rising popularity and availability of rapid prototyping devices such as $3 \mathrm{D}$ printers, it is likely that we will soon experience a shift from mass manufacturing to personalized design and fabrication. In order to maximize the impact of this technology we need computational design methods that allow the appearance, deformation behaviors and motions of fabricated objects to be specified as intuitively and precisely as when creating assets for virtual worlds. The methods described in this paper are aimed at starting to address this challenge.

Computational design tools also have the potential of significantly impacting other research fields. For instance, while humans and animals are composed of both rigid and elastic tissues, most robotic platforms introduced to date are entirely rigid. This simplifies the design and mathematical modeling of the robots, but it also limits their ability to function with skill and purpose in the real world. This significant shortcoming might be avoided by concurrently optimizing the mechanical structure of the robots, the distribution of elastic materials, the placement and characteristics of actuators and appropriate control strategies. At some point in the future, computational design and control methods like the ones described in this paper have the potential of allowing anyone to design and 3D print personalized robots capable of motions as complex and compelling as those of virtual characters.

\section{References}

B ÄCher, M., Bickel, B., James, D. L., AND Pfister, H. 2012. Fabricating articulated characters from skinned meshes. In ' 12.

Calì, J., Calian, D., Amati, C., Kleinberger, R., Steed, A., KAUTZ, J., AND WEYRICH, T. 2012. 3D-printing of nonassembly, articulated models. In ' 12 .

Coros, S., Beaudoin, P., And Van de Panne, M. 2010. Generalized biped walking control. ACM Transctions on Graphics 29, 4, Article 130.

Coros, S., Karpathy, A., Jones, B., Reveret, L., And VAN DE PANNE, M. 2011. Locomotion skills for simulated quadrupeds. ACM Transactions on Graphics 30, 4.

Coros, S., Martin, S., Thomaszewski, B., Schumacher, C., Sumner, R., AND Gross, M. 2012. Deformable objects alive! ACM Trans. Graph. 31, 4 (July), 69:1-69:9. 
Coros, S., Thomaszewski, B., Noris, G., Sueda, S., ForBerG, M., Sumner, R. W., MATusik, W., AND Bickel, B. 2013. Computational design of mechanical characters. ACM Trans. Graph. 32, 4 (July), 83:1-83:12.

Gehring, C., Coros, S., Hutter, M., Bloesch, M., Hoepflinger, M., AND Siegwart, R. 2013. Control of dynamic gaits for a quadrupedal robot. In Proc. of the IEEE International Conference on Robotics and Automation (ICRA).

Hutter, M., Gehring, C., Bloesch, M., Hoepflinger, M., REMY, C., AND Siegwart, R. 2012. Starleth: A compliant quadrupedal robot for fast, efficient, and versatile locomotions. In International Conference on Climbing and Walking Robots (CLAWAR).

Prévost, R., Whiting, E., Lefebvre, S., And SorkineHoRnUnG, O. 2013. Make It Stand: Balancing shapes for 3D fabrication. ACM Transactions on Graphics (proceedings of ACM SIGGRAPH) 32, 4, 81:1-81:10.

Skouras, M., Thomaszewski, B., Coros, S., Bickel, B., AND GRoss, M. 2013. Computational design of actuated deformable characters. ACM Trans. Graph. 32, 4 (July), 82:1$82: 10$. 